\title{
Analytical Characterization of Oscillon Energy and Lifetime
}

\author{
Marcelo Gleisel* and David Sicilia \\ Department of Physics and Astronomy, Dartmouth College, Hanover, NH 03755, USA
}

(Dated: November 20, 2018)

\begin{abstract}
We develop an analytical procedure to compute all relevant physical properties of scalar field oscillons in models with quartic polynomial potentials: energy, radius, frequency, core-amplitude, and lifetime. We compare our predictions to numerical simulations of models with symmetric and asymmetric double well potentials in three spatial dimensions, obtaining excellent agreement. We also explain why oscillons have not been seen to decay in two spatial dimensions.
\end{abstract}

\section{INTRODUCTION}

It is well known that a real scalar field under the influence of certain nonlinear potentials supports long-lived, spatially-localized, time-dependent configurations known as oscillons [1, 2]. Even though there are no topological or nontopological conserved charges associated with such objects, they exhibit remarkably long lifetimes, far exceeding naive estimates from linear approximations to the theory 3. This property has prompted much interest in studying oscillons in more detail in two [4, 5] and three [6] spatial dimensions. Structures with similar qualitative behavior have been observed in many different physical systems, from vibrating grains [7] to acoustic oscillations in the sun 8 .

In the past two years, it has been shown that oscillons are not limited to real scalar field models; they have been found in 2d Abelian-Higgs models [9], and in the Standard Model [10]. They may also play a role in cosmology [11, possibly having an impact on the dynamics of inflationary reheating, in symmetry-breaking transitions, and in false vacuum decay [12. In $1 d$ de Sitter models, oscillons not only form from near-thermal initial conditions, but survive to retain approximately $50 \%$ of the total energy of the universe 13 .

So far, most oscillon properties, such as their energy, radius, and lifetime, have been obtained numerically. It has been shown that they have a near-constant energy and a near-periodic motion with frequency just below the mass of vacuum excitations [5, 14]. In spite of this progress, it is fair to say that a proper analytical understanding of oscillons is still lacking.

In the present work, we will derive all physically relevant properties of scalar oscillons in quartic polynomial potentials. Starting from a simple ansatz for their profile, we develop a nonperturbative analytical procedure to obtain their energy, lifetime, and minimum and maximum values of their frequency, radius, and core amplitude. We compare our analytical results to numerical simulations obtaining excellent agreement.

\section{GENERAL FRAMEWORK}

We begin with the Lagrangian for a $d$-dimensional, spherically-symmetric real scalar field in flat spacetime,

$$
L=c_{d} \int r^{(d-1)} d r\left(\frac{1}{2} \dot{\phi}^{2}-\frac{1}{2}\left(\frac{\partial \phi}{\partial r}\right)^{2}-V(\phi)\right)
$$

where a dot denotes a time derivative, and $c_{d}=$ $2 \pi^{d / 2} / \Gamma(d / 2)$. Inspired by the numerical solutions, which show that an oscillon is well approximated by a Gaussian undergoing near-periodic motion in its amplitude and with an effective radius oscillating with small amplitude about a mean value 2, 3, we approximate the oscillon solution as

$$
\phi(t, r)=A(t) \exp \left(-\frac{r^{2}}{R^{2}}\right)+\phi_{v}
$$

where $A(t) \equiv \phi(r=0, t)-\phi_{v}$ is the displacement from the vacuum, $\phi_{v}$. This approximation works particularly well in $d=2$ and $d=3$, although it clearly fails to reproduce the oscillon's large $r$ behavior, where $\phi(r) \sim \exp [-m r]$.

Since oscillons have been observed in a diversity of quartic polynomial potentials, we write $V(\phi)$ as [15]

$$
V(\phi)=\sum_{j=1}^{4} \frac{g_{j}}{j !} \phi^{j}-V\left(\phi_{v}\right),
$$

where the $g_{j}$ 's are constants and $V\left(\phi_{v}\right)$ is the vacuum energy. Substituting eqs. 2 and 3 in eq. 1 .

$$
L=\left(\frac{\pi}{2}\right)^{\frac{d}{2}} R^{d}\left[\frac{1}{2} \dot{A}^{2}-V(A)\right]
$$

with

$$
V(A)=\frac{1}{2} \omega_{0}^{2} A^{2}+\sum_{n=3}^{4}\left(\frac{2}{n}\right)^{d / 2} \frac{1}{n !} V^{n}\left(\phi_{v}\right) A^{n},
$$

where $V^{n}\left(\phi_{v}\right) \equiv \partial^{n} V\left(\phi_{v}\right) / \partial \phi^{n}$ and $\omega_{0}^{2} \equiv V^{\prime \prime}\left(\phi_{v}\right)+d / R^{2}$ is the linear frequency. (Primes will also denote partial derivatives with respect to $\phi$.) For potentials with $V^{\prime \prime}\left(\phi_{v}\right)>0, V(A)$ is the potential energy of a nonlinear oscillator with at least one equilibrium point (at $A=0$ ) 
for any value of $R$. For conservative motion we can write the energy as,

$$
E=\left(\frac{\pi}{2}\right)^{\frac{d}{2}} R^{d}\left[\frac{1}{2} \dot{A}^{2}+V(A)\right]=\left(\frac{\pi}{2}\right)^{\frac{d}{2}} R^{d} V\left(A_{\max }\right),
$$

where $A_{\max }$ is the positive turning point $\left(A_{\max }>0\right)$ of $A(t)$.

We also write the expression for the frequency $\omega$ associated with the conservative motion between the two turning points,

$$
\frac{2 \pi}{\omega}=T=\int_{0}^{T} d t=2 \int_{A_{\min }}^{A_{\max }} \frac{d A}{\dot{A}},
$$

where $T$ is the period of oscillation, $\dot{A}=$ $\left[2 E / c_{R}-2 V(A)\right]^{\frac{1}{2}}, \quad c_{R} \equiv(\pi / 2)^{d / 2} R^{d}$, and $A_{\min }$ is related to $A_{\max }$ by $V\left(A_{\min }\right)=V\left(A_{\max }\right)$.

It was noted in [3, 15] that the location of $A_{\max }$ relative to the inflection points of $V(A)$ is an accurate indicator of oscillon existence. This can be understood by noting that since the inflection points separate regions of opposite curvature in $V(A)$, the particular region probed by $A_{\max }$ indicates the degree to which the field configuration experiences the stabilizing nonlinearities of the potential $V(\phi)$. Following [15, we therefore adopt $V^{\prime \prime}\left(A_{\max }\right)$ as an indicator of oscillon stability: increasingly negative(positive) values lead to increased stability(instability). Defining $I\left(A_{\max }, R\right) \equiv V^{\prime \prime}\left(A_{\max }\right)$,

$$
I\left(A_{\max }, R\right)=\omega_{0}^{2}+\sum_{n=3}^{4}\left(\frac{2}{n}\right)^{d / 2} \frac{1}{(n-2) !} V^{n}\left(\phi_{v}\right) A_{\max }^{n-2} .
$$

In figure 1 we show the (parabolic) level curve corresponding to $I\left(A_{\max }, R\right)=0$. Since values inside (outside) the parabola have $I<0(I>0)$, it is easy to show that, for a given $R$, there exists a stable region provided that $R>R_{\min }$ where [15]

$$
R_{\min }^{2}=d\left[\frac{1}{2}\left(\frac{2^{3 / 2}}{3}\right)^{d} \frac{\left(V^{\prime \prime \prime}\right)^{2}}{V^{I V}}-V^{\prime \prime}\right]^{-1} .
$$

As the figure indicates, when $R=R_{\min }$, there is only one value of $A_{\max }$ located in the stable region, given by $\bar{A}_{0}=-(4 / 3)^{d / 2} V^{\prime \prime \prime} / V^{I V}$.

\section{LIFE STORY OF AN OSCILLON}

In figure 1, the continuous and dashed lines show curves of constant energy (eq. 6) as a function of $R$ and $A_{\max }$ for a symmetric double-well potential,

$$
V(\phi)=\phi^{2}-\phi^{3}+\phi^{4} / 4
$$

that is, $g_{1}=0, g_{2}=2, g_{3}=-6, g_{4}=6, \phi_{v}=0$ in eq. 5. [Quantities were made dimensionless with

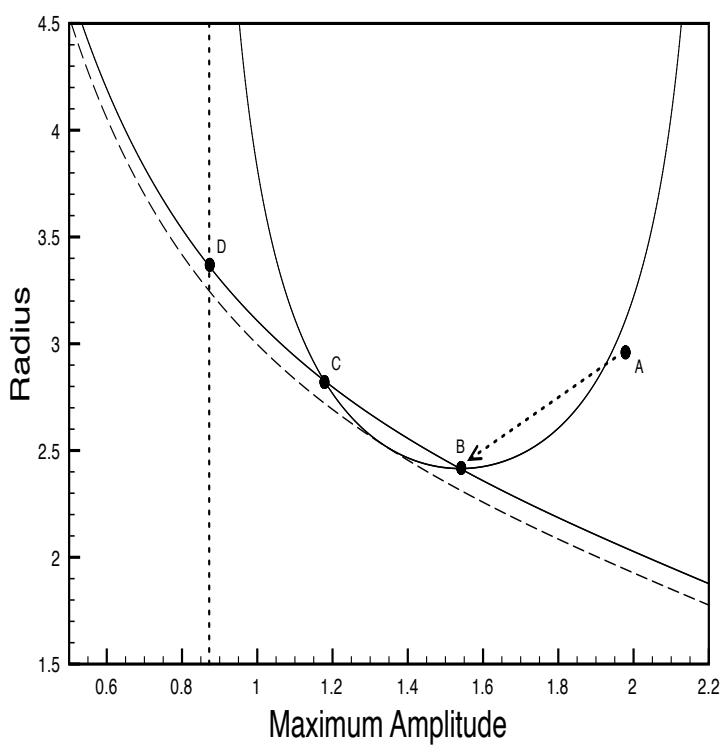

FIG. 1: Two curves of constant energy, $E_{\text {osc }}=41.3$ (continuous line), and $E_{\text {attract }}=37.7$ (dashed line), together with the parabolic level curve $I\left(A_{\max }, R\right)=0$. Vertical dashed line locates the asymptote of $I=0$.

$x^{\mu}=x^{\mu}\left(\sqrt{g^{4}} \phi_{v}\right)^{-1}$ and the primes have been suppressed.] We also plot the parabola $I\left(R, A_{\max }\right)=0$ marking the boundary between the stable region $(I<0$, above the curve) and the unstable region $(I>0$, below the curve). Note that the presence of an oscillon in the unstable region does not necessarily mean that it will immediately decay; the degree of stability is given roughly by the vertical distance of a point to the curve $I=0$.

Using the curve $I=0$ in fig. 1, we can describe the trajectory modeling the onset of an oscillon from an initial configuration starting at a somewhat arbitrary point $A$. On its way to an oscillon, the configuration will radiate excess energy until reaching point $B$. To determine the location of point $B$, note that the onset of the oscillon phase is marked by a minimum radius. (In what follows it will be clear that this must be true if radiation radically slows down.) Taking the minimum radius given by eq. 9, and using that the oscillon stage should begin in the stable region, fixes the location of point $\mathrm{B}$ to $A=\bar{A}_{0}$ and $R=R_{\min }$, as shown in figure 1 As we shall see, this is confirmed by numerical results. For the potential of eq. 10, $R_{\text {min }}=2.42$ and $A_{B}=\bar{A}_{0}=(4 / 3)^{3 / 2}=1.54$, in excellent agreement with the inset in figure 2

Since our model approximates an oscillon as having constant energy, we input these values into eq. 6, to obtain the oscillon energy, $E_{\mathrm{osc}}$ :

$$
\begin{aligned}
E_{\mathrm{osc}} \simeq & E\left(A_{B}, R_{B}\right)=\left(\frac{\pi}{2}\right)^{d / 2} R_{B}^{d} V\left(A_{B}, R_{B}\right)= \\
& \frac{1}{8}\left(\frac{\pi}{2}\right)^{d / 2}\left(\frac{8 \sqrt{2}}{9}\right)^{d} \frac{\left(V^{\prime \prime \prime}\right)^{4}}{\left(V^{I V}\right)^{3}} R_{B}^{d}=41.3 .
\end{aligned}
$$




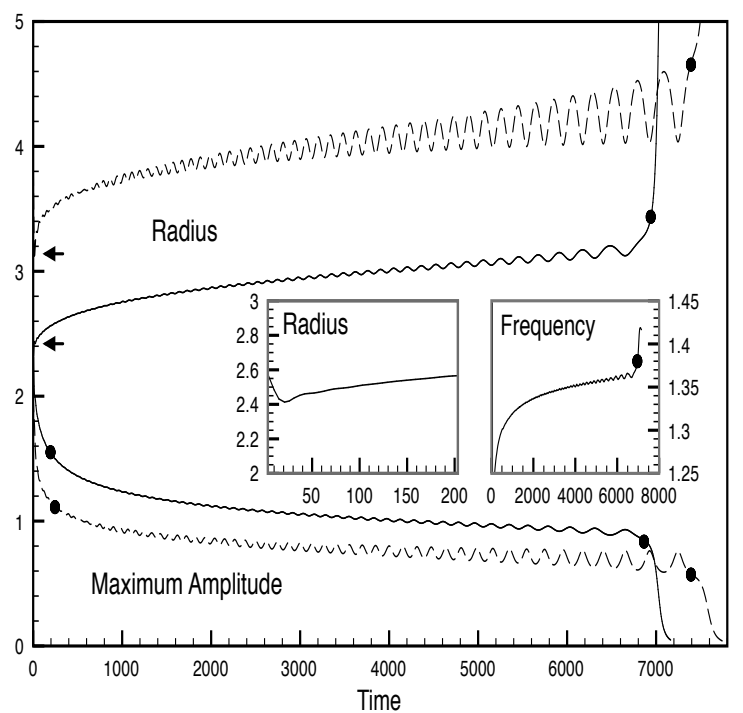

FIG. 2: The maximum amplitude and radius as a function of time for a configuration with $R_{0}=2.86$ and $A_{0}=2$ in the symmetric double well of eq. 10 (continuous lines), and for an asymmetric double well $\left[V(\phi)=\frac{1}{2} \phi^{2}-\frac{2.16}{3} \phi^{3}+\frac{1}{4} \phi^{4}\right]$ with $A_{0}=2$ and $R_{0}=4$ (dashed lines). The analytical predictions at the points $\left(A_{B}, R_{B}\right)$ and $\left(A_{D}, R_{D}\right)$ are indicated by arrows and dots. The insets show the minimum radius and frequency for the symmetric double well.

where the numerical value given is for $d=3$ and the potential of eq. 10. Compare with numerical results 2, 3], where, roughly, $42 \leq E_{\text {osc }} \leq 45$ during the oscillon's lifetime.

In addition to the above estimate for $E_{\text {osc }}$, we can derive an absolute lower bound for the oscillon energy. This is found by noting that the dashed line in fig. 1 shows a critical energy, denoted $E_{\text {attract }}$, below which the energy curves do not probe the stable region. Using the $I=0$ condition, one can eliminate $R$ from the expression of the energy (eq. 6), which becomes a function only of $A_{\max }$. Remarkably, this function has a minimum, the asymptote energy of figure 1. For the potential of eq. 10 . $E_{\text {attract }}=37.7(d=3)$ and $E_{\text {attract }}=4.44(d=2)$.

Having arrived at point $B$ (after radiating excess initial energy) the oscillon proceeds leftward along its line of constant energy, $E_{\text {osc }}$ (Traveling rightward would cause $R$ to tend to zero, which is unphysical). When the oscillon passes point $C$, its distance from $I=0$ begins to increase and its instability grows. To estimate the point of oscillon decay we note that, at point $D$, the vertical distance to the $I=0$ curve becomes infinite. Mathematically, point $D$ is found by taking the limit $R \rightarrow \infty$ in the expression $I=0$ and solving for $A_{D}$, the decay amplitude. For a cubic potential this gives, $A_{D}=-(3 / 2)^{3 / 2}\left(V^{\prime \prime} / V^{\prime \prime \prime}\right)$. For a quartic potential,

$$
A_{D}=A_{B}\left[1-\left(1-2\left(\frac{3 \sqrt{2}}{4}\right)^{d} \frac{V^{I V} V^{\prime \prime}}{V^{\prime \prime \prime 2}}\right)^{1 / 2}\right] \simeq 0.84
$$

where $A_{B}$ was obtained above and the numerical result is for the potential of eq. 10 in $d=3$.

To obtain the final radius $R_{D}$, we evaluate eq. 11 at point $D: \quad E_{\mathrm{osc}}=(\pi / 2)^{d / 2} R_{D}^{d} V\left(A_{D}, R_{D}\right)$. Solving for $R_{D}$

$$
g\left(A_{D}\right) R_{D}^{d}+\left(\frac{d}{2} A_{D}^{2}\right) R_{D}^{d-2}-E_{\text {osc }}\left(\frac{2}{\pi}\right)^{d / 2}=0,
$$

where $g\left(A_{D}\right) \equiv V^{\prime \prime} A_{D}^{2} / 2+\sum_{n=3}^{4}(2 / n)^{d / 2} V^{n} A_{D}^{n} / n$ !. In $d=3$, one gets a cubic equation for $R_{D}$. For the potential of eq. 10 , with $E_{\mathrm{osc}}=41.3$ and $A_{D}=0.84$, we obtain, $R_{D}=3.43$. In figure 2 , we compare numerical and analytical values for $\left(A_{B}, R_{B}\right)$ (left arrows and dots) and $\left(A_{D}, R_{D}\right)$ (right dots) for this potential (continuous lines) and for an asymmetric potential (dashed lines). [The numerical simulation measures $R$ by fitting a Gaussian to the oscillon and reading off the radius.]

Since we know $A_{\max }(t)$ throughout the oscillon's life, we can use the potential $V(A)$ to compute $A_{\min }$ and then use eq. 7 to obtain $\omega(t)$. For example, using $A_{\max }=$ $A_{D}$ gives the decay (or critical) frequency, $\omega_{D}=\omega_{\text {crit }}=$ $1.38<\omega_{\text {mass }}=V^{\prime \prime}\left(\phi_{v}\right)=\sqrt{2}$. In the inset of figure 2 we compare analytical (dot) and numerical values. It should be noted that this model correctly predicts that the oscillon frequency tends to a maximum (as observed in, for example, [14) as it approaches decay.

\section{OSCILLON LIFETIME}

Due to the remarkable near-periodicity of an oscillon, its spectral function in frequency space displays a series of narrow peaks, with the dominant one just below the mass frequency [5]. Only a small radiating tail penetrates the region with $\omega>\omega_{\text {mass. }}$ As in ref. [5, we model the power spectrum of the oscillon core amplitude $\phi(0, t)$ around its dominant frequency peak with a Breit-Wigner profile. Since the power is proportional to the square of the amplitude of a given frequency component, we write

$$
a^{2}(\omega)=K\left[\left(\frac{\omega-\omega_{\mathrm{osc}}}{\frac{1}{2} \Gamma}\right)^{2}+1\right]^{-1}
$$

where $a^{2}(\omega)$ is the square of the amplitude of the frequency component of $\phi(0, t)$ with frequency $\omega$, and $K$ is a constant to be determined. Given that the amplitude of this primary frequency peak is approximately that of the oscillon itself (because the first peak is narrow and holds most of the power) $a^{2}\left(\omega_{\mathrm{osc}}\right)=K \approx A_{\max }^{2}$, the oscillon's 
core amplitude. Since the constant $\Gamma$ setting the width of the resonance peak is related to the (inverse) timescale associated with the instantaneous radiation rate of the oscillon, it is natural to let $\Gamma=\frac{d \dot{E}}{d E}$, where $E$ is the oscillon energy.

Most of the (small-amplitude) radiation leaking from the oscillon is contained in a mode with wavelength commensurate with the size of the oscillon - that is, with wavelength $\sim 4 R$. The frequency of this wave is therefore $\omega_{\text {rad }}=\sqrt{\omega_{\text {mass }}^{2}+(2 \pi / 4 R)^{2}}$.

Now consider an infinitesimally thin shell of radius $R$ around the oscillon with volume $c_{d} R^{d-1} d r$, which is filled with the outgoing radiation wave $\left(\omega=\omega_{\text {rad }}\right)$ traveling with speed $v_{\text {rad }}$. Within this thin shell, the wave can be approximated as a $1 d$ plane wave of constant amplitude; hence its energy density is $A_{\text {rad }}^{2} \omega_{\text {rad }}^{2} / 2$, where $A_{\text {rad }}$ is the amplitude of the radiation wave in the shell. Since $A_{\mathrm{rad}}^{2}$ is given by $a^{2}\left(\omega_{\mathrm{rad}}\right)$, the total radiation energy contained in the thin shell is $a^{2}\left(\omega_{\mathrm{rad}}\right) \frac{\omega_{\mathrm{rad}}^{2}}{2} c_{d} R^{d-1} d r$. The radiation travels outward at a speed $v_{\text {rad }}=\omega_{\text {rad }} / k_{\text {rad }}$, and $d r=$ $v_{\text {rad }} d t$. The amount of energy lost by the oscillon per unit time, $-\dot{E}$, is therefore

$$
-\dot{E}=A^{2} \frac{1}{2} \omega_{\mathrm{rad}}^{2} c_{d} R^{d-1} v_{\mathrm{rad}}\left[\left(\frac{\omega_{\mathrm{rad}}-\omega_{\mathrm{osc}}}{\frac{1}{2} \frac{d \dot{E}}{d E}}\right)^{2}+1\right]^{-1} .
$$

If the oscillon is long lived, $\omega_{\text {rad }}-\omega_{\text {osc }} \gg d \dot{E} / d E$. In this case, and writing $\frac{d \dot{E}}{d E}=\frac{d \dot{E}}{d t} \frac{1}{\dot{E}}$, we have

$$
\ddot{E}^{2}+4 \alpha \dot{E}^{3}=0
$$

where $\alpha \equiv 2\left(1-\omega_{\text {osc }} / \omega_{\text {rad }}\right)^{2}\left[A^{2} c_{d} R^{d-1} v_{\text {rad }}\right]^{-1}$.

To integrate eq. 16, we take $\alpha$ to be constant, a good approximation for an oscillon. Then,

$$
E(t)=\frac{E_{i}-E_{\infty}}{\alpha\left(E_{i}-E_{\infty}\right) t+1}+E_{\infty},
$$

where $E_{\infty}$ is the asymptotic energy as $t \rightarrow \infty$ and $E_{i}$ the initial energy. The oscillon decays at energy $E_{D}>E_{\infty}$ in a time $\tau_{\text {life }}$ given by

$$
\tau_{\text {life }}=\frac{1}{\alpha}\left(\frac{1}{E_{D}-E_{\infty}}-\frac{1}{E_{i}-E_{\infty}}\right) .
$$

If $E_{i} \sim E_{D}$, the lifetime will be approximately zero, as expected. When $E_{i} \gg E_{D}$, the lifetime becomes independent of $E_{i}$ and tends to a maximum, $\tau_{\text {maxlife }} \simeq \frac{1}{\alpha} \frac{1}{E_{D}-E_{\infty}}$. To evaluate this expression, note that $E_{D}$ is simply the plateau energy obtained in eq. 11. For $E_{\infty}$, being the minimum energy the oscillon may possess, it is most natural to choose $E_{\infty}=E_{\text {attract }}$. For the other parameters in $\alpha$, we analytically calculate the average of each quantity over the stable phase of the oscillon's life $(C \rightarrow D$ in figure 11. For the potential $V(\phi)=\phi^{2}-\phi^{3}+\frac{1}{4} \phi^{4}$, we obtain $\tau_{\text {maxlife }} \approx 6300$, which is quite accurate.

Finally, note that eq. 16 admits the solution, $\dot{E}=$ $-\alpha\left(E-E_{\text {attract }}\right)^{2}$. Thus, if $E \rightarrow E_{\text {attract }}, \dot{E} \rightarrow 0$. In $d=2$, one can show numerically that the lowest energy oscillon has $E_{\text {osc }}=E_{\text {attract }}=4.44$. We thus see why $\tau_{\text {maxlife }} \rightarrow \infty$ in $d=2$ : the oscillon reaches its attractor value and stops radiating.

We have analytically computed all relevant physical properties of scalar field oscillons in quartic polynomial potentials, confirming that oscillons are indeed attractors in field configuration space and that their lifetimes can be estimated by studying their main radiating mode. Our results suggest why $d=2$ oscillons seem to be stable: they reach their attractor state before decaying. We intend to extend our approach to oscillons in models with gauge fields and with more complicated potentials. This work was partially supported by a National Science Foundation grant PHY-0653341.

* Electronic address: gleiser@dartmouth.edu

$\dagger$ Electronic address: davidovich@dartmouth.edu

[1] I. L. Bogolubsky and V. G. Makhankov, JETP Lett. 24, 12 (1976) [Pis'ma Zh. Eksp. Teor. Fiz. 24 (1976) 15].

[2] M. Gleiser, Phys. Rev. D 49, 2978 (1994).

[3] E. J. Copeland, M. Gleiser and H.-R. Müller, Phys. Rev. D 52, 1920 (1995).

[4] M. Gleiser and A. Sornborger, Phys. Rev. E 62, 1368 (2000).

[5] M. Hindmarsh and P. Salmi, Phys. Rev. D 74, 105005 (2006); 77, 105025 (2008).

[6] E. Honda and M. Choptuik, Phys. Rev. D 65, 084037 (2002); G. Fodor, P. Forgács, P. Grandclément, and I. Rácz, Phys. Rev. D 74, 124003 (2006); V. A. Koutvistsky and E. M. Maslov, J. Math. Phys. 47, 022302 (2006).

[7] P. Umbanhowar, F. Melo, and H. Swinney, Nature 382, 793 (1996); L. S. Tsimring and S. Aranson, Phys. Rev. Lett. 79, 213 (1997).

[8] O. M. Umurhan, L. Tao, and E. A. Spiegel, Ann. N. Y. Acad. Sci. 867, 298 (1998).

[9] M. Gleiser and J. Thorarinson, Phys. Rev. D 76, 041701(R) (2007).

[10] E. Farhi, N. Graham, V. Khmeani, R. Markov, and R. Rosales, Phys. Rev. D 72, 101701 (2005); N. Graham, Phys. Rev. Lett. 98, 101801 (2007), [Erratum-ibid. 98, 189904 (2007)].

[11] M. Gleiser, Int. J. Mod. Phys. D 16, 219 (2007).

[12] M. Gleiser, B. Rogers, and J. Thorarinson, Phys. Rev. D 77, 023513 (2008); M. Gleiser and R. Howell, Phys. Rev. Lett. 94, 151601 (2005)

[13] E. Farhi et al., Phys. Rev. D 77, 085019 (2008).

[14] P. M. Saffin and A. Tranberg, JHEP 0701, 030 (2007).

[15] M. Gleiser, Phys. Lett. B 600, 126 (2004). 